González-De Los Reyes, Y.; Fernández-Ortega, J. y Garavito-Peña, F. (2019) Características de fuerza y velocidad de ejecución en mujeres jóvenes futbolistas. Characteristics of Strength and Speed of Execution in Young Women Soccer Players. Revista Internacional de Medicina y Ciencias

de la Actividad Física y el Deporte vol. 19 (73) pp. 167-179 Http://cdeporte.rediris.es/revista/revista73/artcaracteristicas1009.htm DOI: http://doi.org/10.15366/rimcafd2019.73.012

\title{
ORIGINAL
}

\section{CARACTERÍSTICAS DE FUERZA Y VELOCIDAD DE EJECUCIÓN EN MUJERES JÓVENES FUTBOLISTAS}

\section{CHARACTERISTICS OF STRENGTH AND SPEED OF EXECUTION IN YOUNG WOMEN SOCCER PLAYERS}

\author{
González-De Los Reyes, Y.'; Fernández-Ortega, J. ${ }^{2}$ y Garavito-Peña, F. ${ }^{3}$ \\ 1 Doctora en ciencias de la actividad física y del deporte, Grupo de Investigación Cuerpo, Sujeto y \\ Educación, Universidad Santo Tomás, sede Bogotá, D.C., Facultad de Cultura Física, Deporte y \\ Recreación. Bogotá, D.C. (Colombia) yennysgonzalez@usantotomas.edu.co \\ 2 Doctor en ciencias de la actividad física y del deporte. Grupo Centro de Investigaciones en Actividad \\ física y deporte CIDER. Universidad Pedagógica Nacional. Bogotá, D.C (Colombia) \\ jairofdz@pedagogica.edu.co \\ ${ }^{3}$ Magister en fisiología y cultura física terapéutica. Grupo de Investigación GICADES, Universidad \\ Santo Tomás, sede Bogotá, D.C., Facultad de Cultura Física, Deporte y Recreación. Bogotá, D.C., \\ (Colombia) felipegaravito@usantotomas.edu.co
}

AGRADECIMIENTOS Y/O FINANCIACIÓN: Al fondo de desarrollo de la investigación FODEIN de la Universidad Santo Tomás, Bogotá, D.C. (Colombia) y a la Universidad Pedagógica Nacional, Bogotá, D.C. (Colombia)

Código UNESCO / UNESCO code: 320000 Ciencias de la salud / Health Sciences. Clasificación del Consejo Europa / Council of Europe classification: 17.Otras: Actividad física y Deporte / Physical Activity and Sport.

Recibido 2 de mayo de 2017 Received May 2, 2017

Aceptado 22 de enero de 2018 Accepted January 22, 2018

\section{RESUMEN}

El objetivo de este estudio fue caracterizar y comparar la fuerza máxima y rápida, la potencia anaeróbica, la velocidad de ejecución y de desplazamiento en función de la posición de juego en 59 jóvenes futbolistas distribuidas en dos categorías. La metodología consistió en una valoración de masa corporal, talla y 4 pruebas, fuerza explosiva (CMJ Y SJ), velocidad $(30 \mathrm{~m})$, la potencia anaeróbica (Prueba de Wingate) y la fuerza máxima (\%1RM). Los resultados mostraron a las 
pre-juveniles con los mejores registros en la mayoría de variables a excepción de la talla y el VMP sentadilla. En la prueba de potencia máxima las porteras y defensas pre-juveniles obtuvieron el mejor registro $(409,11 \mathrm{~W} \pm 86,73 \mathrm{~W})$ y $1 \mathrm{RM}(60,58$ $\mathrm{Kg} \pm 13,69 \mathrm{Kg}$ ) sin diferencias significativas. Finalmente, se encontró una interacción significativa entre la posición y la categoría de juego en VMP sentadilla, F55=21,41; $\mathrm{p}=0,021$, eta cuadrado $=0,093$ entre las jugadoras de las dos categorías estudiadas.

PALABRAS CLAVE: Fuerza, 1RM, futbol femenino, potencia anaeróbica, velocidad de ejecución y velocidad.

\begin{abstract}
The objective of this study was to characterize and compare the maximum and fast strenght, anaerobic power, speed of execution and displacement according to the playing position in 59 young players divided into two categories. The methodology consisted of an assessment of body mass, height and 4 tests, explosive force (CMJ and SJ), velocity $(30 \mathrm{~m})$, anaerobic power (Wingate test) and maximum force (\% 1RM). The results showed the under 15 years old with the best records on most variables except for the size and mean propulsive velocity (MPV) squat. In the maximum power test pre-juvenile goalkeepers and defences line obtained the best record $(409.11 \mathrm{~W} \pm 86.73 \mathrm{~W})$ and $1 \mathrm{RM}(60.58 \mathrm{~kg} \pm 13.69 \mathrm{Kg})$ without significant differences. Finally, we found a significant interaction between the position and the game category in MPV squat, F55 = 21.41; $\mathrm{P}=0.021$, eta square $=0.093$ among the players of the two categories studied.
\end{abstract}

KEYWORDS: Strength, 1RM, female soccer, anaerobic power, speed of execution and speed.

\title{
INTRODUCCIÓN
}

El fútbol femenino es una disciplina deportiva con un gran aumento en su popularidad y profesionalismo, sin embargo, los estudios a nivel de características de condición física y regímenes de entrenamiento son aún muy limitados, en adolescentes y mujeres colombianas. La actividad física se manifiesta en el fútbol por la sucesión de esfuerzos cortos intercalándose con periodos de trabajo de intensidad moderada y baja y pausas de recuperación (Stolen et al., 2005). Por esta razón vemos como las jugadoras elite han aumentado las carreras de alta intensidad en un $28 \%$ y los sprint en un $24 \%$ más que las jugadoras de nivel moderado (Datson et al., 2014).

Adicionalmente a los aspectos técnicos y tácticos, la fuerza, la potencia la velocidad, la agilidad y la resistencia aeróbica son aspectos fundamentales en el rendimiento de los futbolistas. (Impellizzeri et al., 2008) (Mujika et al., 2009). De esta manera, el incremento en la fuerza muscular de los músculos del tren inferior 
permite disponer de una mejor aceleración y velocidad para la técnica del dribling, los cambios de dirección y de velocidad que son fundamentales en el futbol (Suleyman et al., 2010).

La velocidad que se requiere entonces al momento de ir al ataque o contraatacar pone de manifiesto la necesidad de contar con jugadores no solamente veloces, sino también fuertes. Por lo tanto, la preparación física en el fútbol, tanto en el de alto rendimiento, como en mujeres infantiles y pre-juveniles que son las que nos ocupa en este estudio, le otorga gran importancia al hecho de desarrollar adecuados niveles de potencia. El objetivo primordial de los técnicos de fútbol es formar futbolistas potentes, es decir que combinen adecuadamente la velocidad y la fuerza.

Además, la fuerza marcara la diferencia en la realización de la técnica, que muchas veces no se debe a la carencia del desarrollo de las capacidades coordinativas, sino a la falta de trabajo de los grupos musculares que intervienen en forma directa y eficaz en un gesto deportivo. Entre los objetivos de la evaluación de la fuerza queremos destacar la importancia en el rendimiento en un deporte específico, partiendo de la caracterización del esfuerzo y su aplicación en la programación del entrenamiento. El poder emplear altos niveles de fuerza y potencia en los deportistas, usualmente se relacionan con una mayor capacidad para acelerar el propio peso corporal y objetos externos empleados en diversos deportes (Berger, 1982). Por consiguiente, es lógico pensar que el entrenamiento de esta capacidad aumente la velocidad en condiciones similares a las competiciones. Además, el nivel de relación entre la fuerza y la velocidad depende en gran medida de la valoración de la fuerza y de su desarrollo. Por otra parte, la relación planteada entre los incrementos de fuerza mediante el proceso del entrenamiento y los incrementos de velocidad dependerán entonces del nivel de especificidad de la velocidad de dicho entrenamiento.

Otra cualidad estrechamente relacionada con la fuerza es la velocidad de ejecución, y dicha relación aumenta si se incrementa la resistencia a mantener o vencer. Al aumentar la velocidad en la ejecución de la fuerza podrá producir una potencia mayor, lo cual se manifestará en una velocidad mayor en el momento de ejecución de un gesto deportivo (González, 1997).

En relación con las características de los desplazamientos que se realizan en el fútbol, y en lo que respecta específicamente al fútbol femenino, estudios de Hewitt et al., (2007) registran este recorrido en un partido en $9.140 \mathrm{~m}$ y Scott y Drust (2007) en $11.979 \mathrm{~m}$. Ya hablando específicamente de los desplazamientos de alta intensidad, D'Ottavio (1998) concluye en su estudio que las duraciones de los desplazamientos a la máxima intensidad casi nunca son superiores a los 3 y 4 segundos, lo que supone una distancia comprendida entre 15 y $25 \mathrm{~m}$. Señala, además, que este tipo de acciones se repiten entre 60 y 70 veces a lo largo de un partido. Mohr et al., (2003) en su estudio aseveran que, durante un partido, una acción de sprint ocurre cada 90 segundos, con una duración entre 2 y 4 s. 
En la actualidad es de igual importancia el incremento de la distancia total recorrida como el incremento de las acciones explosivas realizadas en el transcurso de un partido. Todd et al., (2002) nos muestran que entre las acciones más características que se dan en cualquier partido de fútbol podemos destacar la puesta en acción o arranque, el frenado, los saltos, las cargas, los golpeos, los remates, los tiros y las entradas. Las acciones anteriormente mencionadas se relacionan con el factor determinante de la fuerza. Finalmente, un reciente estudio que analiza el comportamiento de jugadoras en competiciones internacionales observa un déficit significativo en la condición física, fundamentalmente de naturaleza anaeróbica (Andersson, Krustrup, y Mohr, 2007). La valoración de las demandas físicas de las diferentes posiciones de juego es esencial para asegurar la especificidad y la identificación potencial de talentos de los jugadores en especial de la rama femenina.

\section{OBJETIVO}

Caracterizar y comparar la fuerza máxima y explosiva, la potencia anaeróbica, la velocidad de ejecución y de desplazamiento en función de las categorías y la posición ocupada en el terreno de juego en mujeres jóvenes futbolistas.

\section{MATERIAL Y METODOS}

La población estuvo compuesta por 59 jóvenes futbolistas que de forma voluntaria accedieron a participar en el estudio y fueron distribuidas en dos categorías por edad: Categoría infantil (12-13 años) y categoría pre-juvenil (14-15 años). Inicialmente se realizó una reunión con los entrenadores, jugadoras, y padres de familia, con el fin de informar acerca de los objetivos del estudio, metodología, beneficios y posibles riesgos. Posteriormente, los padres de las jugadoras al ser estas menores de edad firmaron el consentimiento informado. El estudio estuvo avalado por el comité de ética de la Universidad Santo Tomas de Bogotá, Colombia.

Evaluación de la fuerza y potencia: Antes de las evaluaciones y del proceso de entrenamiento las participantes realizaran un proceso de familiarización en el cual movilizaron diferentes cargas a la mayor velocidad. La fuerza máxima concéntrica se obtuvo a partir del método de 1RM en el movimiento de media sentadilla con un ángulo de $90^{\circ}$ y la deportista realizo la extensión los más rápido posibles hasta llegar a $180 \%$. Previo se realizó un calentamiento de cinco repeticiones con cargas del $40 \%-60 \%$ de la escala de percepción del esfuerzo OMNI-RES (Robertson et al., 2003). Para determinar la 1RM se incrementó la carga progresivamente $10 \%$ y $20 \%$ con intervalos de tres minutos de recuperación, de acuerdo con los resultados de la escala OMNI-RES, hasta que la deportista fue incapaz de realizar la extensión a $180^{\circ}$. La carga más alta movilizada cumpliendo esta condición, fue considerada como la fuerza máxima. Para identificar la relación potencia-carga se utilizaron cargas del 30\%,45\%,60\%,70\%,80\%,90\% de $1 \mathrm{RM}$, y se 
le indico a las deportistas que realizarán a la máxima velocidad posible cada uno de los movimientos, de esta forma se obtuvo la máxima velocidad de ejecución (Izquierdo et al., 2002).

La velocidad de carrera se evaluó mediante la prueba de 30 metros en el campo de fútbol (Kotzamanidis et al., 2005). Para el registro de la velocidad de carrera se utilizó el sistema de foto células de luz infrarroja modelo WL34-R240. (Marca Sick ®Potencia de encendido: Relevo, SPDT. Aislado. Max. Frecuencia de encendido: 10/s. Tiempo de demora: tabla, ajustable 0.5 a $10 \mathrm{~s}$.) Las cuales se ubicaron al inicio a los 30 metros y al final del recorrido.

Las participantes realizaron dos tipos de salto el squat jump (SJ) en una posición de flexión de la rodilla a $90^{\circ}$ y el counter movement jump (CMJ) que realizaran el movimiento lo más rápido posible. En ambos casos las deportistas debían tener las manos en la cintura (Kotzamanidis et al., 2005). Para la estimación de la saltabilidad se utilizó el Optojump Microgate ® Alemania (precisión de 1/1000 segundos).

La valoración de la potencia y velocidad máxima de miembros inferiores se realizó a través de dos protocolos: un test en cicloergometro que consiste en pedalear sentado durante 30 segundos a máxima velocidad contra una resistencia constante equivalente al $0,53 \%$ del peso corporal. Se utilizó una Bicicleta Monark 835E (Monarkexercice, Varberg Sweden), ajustando el sillín a la altura de espina lliaca de las participantes.

Previamente se realizaron cinco minutos de calentamiento a 40 RPM, con una carga $0,30 \%$ del peso corporal. En los minutos 2, 3 y 4 se ejecutó un sprint durante cinco segundos, después de tres minutos de recuperación las participantes realizaron la prueba. El segundo protocolo se llevó a cabo mediante la prueba de sentadilla, se realizaron tres series de cinco repeticiones con una carga del $45 \%$ del 1 RM con el propósito de obtener la Velocidad Media Propulsiva (VMP) y la potencia máxima. Durante todo el procedimiento se animó a las deportistas a realizar a máxima velocidad la ejecución. Entre repeticiones se dejaron cinco minutos de recuperación.

Para obtener la velocidad de desplazamiento de la barra (metros segundos) y de la potencia media (vatios) se utilizó un transductor de velocidad lineal (TFORCE Dynamic Measurement System2, Ergotech Consulting S.L., Murcia, España, con velocidad de muestreo de $1000 \mathrm{~Hz}$ y precisión de $0.0002 \mathrm{~m}$ ) que calcula automáticamente los parámetros cinemáticos relevantes de cada repetición y proporciona retroalimentación auditiva y visual de la velocidad en tiempo real. (Sánchez et al., 2014) 
Análisis estadístico

Para el análisis estadístico se utilizó el software IBMSPSS versión 22 (Licencia Universidad Santo Tomás de Bogotá- Colombia). Se realizaron análisis descriptivos para cada variable y se reportó la media y la desviación estándar (SD) para cada una de las posiciones de juego. Posteriormente se utilizó un análisis de varianza (ANOVA) factorial $2 X 2$ para comparar cada variable, de acuerdo, al grupo y la posición de juego. Se empleó un nivel de significancia $p \leq 0,05$.

\section{RESULTADOS}

En la tabla 1. Se presentan los resultados obtenidos de todas las variables analizadas distinguiendo por posiciones de juego, la posición 1 (Porteras y Defensas) y la posición 2 (Volantes y Delanteras) dentro de cada categoría.

\begin{tabular}{|c|c|c|c|c|c|}
\hline & & & INFANTIL & & PREJUVENIL \\
\hline VARIABLE & Posición & $\mathbf{N}$ & Media \pm SD & $\mathbf{N}$ & Media \pm SD \\
\hline \multirow[t]{2}{*}{ Edad (años) } & 1 & 18 & $14,10 \pm 0,39$ & 8 & $16,00 \pm 0,00$ \\
\hline & 2 & 17 & $14,05 \pm 0,52$ & 16 & $16,06 \pm 0,25$ \\
\hline \multirow[t]{2}{*}{ Talla (cm) } & 1 & 18 & $158,83 \pm 8,03$ & 8 & $163,46 \pm 5,21$ \\
\hline & 2 & 17 & $157,70 \pm 5,30$ & 16 & $156,17 \pm 5,73$ \\
\hline \multirow[t]{2}{*}{ Masa Corporal(Kg) } & 1 & 18 & $48,10 \pm 6,60$ & 8 & $55,43 \pm 8,11$ \\
\hline & 2 & 17 & $48,14 \pm 4,61$ & 16 & $54,39 \pm 5,42$ \\
\hline \multirow[t]{2}{*}{ Carrera 30m (s) } & 1 & 18 & $5,10 \pm 0,21$ & 8 & $4,75 \pm 0,16$ \\
\hline & 2 & 17 & $5,07 \pm 0,20$ & 16 & $4,80 \pm 0,21$ \\
\hline \multicolumn{6}{|l|}{ Potencia Wingate } \\
\hline \multirow[t]{2}{*}{ Máxima (vatios) } & 1 & 18 & $250,76 \pm 58,50$ & 8 & $409,11 \pm 86,73$ \\
\hline & 2 & 17 & $252,78 \pm 58,00$ & 16 & $353,20 \pm 103,67$ \\
\hline \multirow[t]{2}{*}{ Máxima Relativa(v/Kg) } & 1 & 18 & $5,16 \pm 0,56$ & 8 & $7,36 \pm 0,70$ \\
\hline & 2 & 17 & $5,23 \pm 0,99$ & 16 & $6,77 \pm 0,97$ \\
\hline \multirow[t]{2}{*}{ Mínima(vatios) } & 1 & 18 & $165,28 \pm 38,88$ & 8 & $237,00 \pm 50,85$ \\
\hline & 2 & 17 & $165,59 \pm 32,70$ & 16 & $220,38 \pm 40,66$ \\
\hline \multirow[t]{2}{*}{ Mínima Relativa(v/Kg) } & 1 & 18 & $3,41 \pm 0,54$ & 8 & $4,25 \pm 0,45$ \\
\hline & 2 & 17 & $3,42 \pm 0,48$ & 16 & $4,06 \pm 0,69$ \\
\hline \multirow[t]{2}{*}{ Salto en contramovimiento $(\mathrm{cm})$} & 1 & 18 & $24,74 \pm 3,31$ & 8 & $25,86 \pm 2,94$ \\
\hline & 2 & 17 & $23,48 \pm 4,28$ & 16 & $25,49 \pm 3,90$ \\
\hline \multirow[t]{2}{*}{ Salto desde sentadilla $(\mathrm{cm})$} & 1 & 18 & $21,21 \pm 2,39$ & 8 & $24,66 \pm 2,98$ \\
\hline & 2 & 17 & $20,67 \pm 4,47$ & 16 & $24,27 \pm 3,39$ \\
\hline \multirow[t]{2}{*}{ Potencia en sentadilla(vatios) } & 1 & 18 & $202,06 \pm 41,18$ & 8 & $225,50 \pm 42,78$ \\
\hline & 2 & 17 & $191,47 \pm 45,24$ & 16 & $216,56 \pm 59,31$ \\
\hline \multirow[t]{2}{*}{ VMP sentadilla $(\mathrm{m} / \mathrm{s})$} & 1 & 18 & $0,85 \pm 0,04$ & 8 & $0,80 \pm 0,12$ \\
\hline & 2 & 17 & $0,86 \pm 0,06$ & 16 & $0,71 \pm 0,09$ \\
\hline Peso VWP (Kg) & 1 & 18 & $24,23 \pm 4,71$ & 8 & $28,95 \pm 4,21$ \\
\hline
\end{tabular}


Rev.int.med.cienc.act.fís.deporte - vol. 19 - número 73 - ISSN: 1577-0354

\begin{tabular}{|lcrrrr|} 
& 2 & 17 & $22,87 \pm 5,31$ & 16 & $31,15 \pm 10,80$ \\
\hline RM alcanzado $(\mathrm{Kg})$ & 1 & 18 & $31,16 \pm 5,11$ & 8 & $60,58 \pm 13,69$ \\
\hline & 2 & 17 & $29,04 \pm 5,09$ & 16 & $57,20 \pm 11,98$ \\
\hline
\end{tabular}

Tabla 1. Resultados en función de las posiciones de juego dentro de cada categoría.

En la tabla 2. Se muestra los resultados obtenidos de todas las variables estudiadas por categoría y por posición de juego.

\begin{tabular}{|c|c|c|c|c|c|c|c|c|}
\hline Variable & Factores & F-Ratio & $\begin{array}{c}\text { Eta } \\
\text { cuadrado } \\
\text { parcial }\end{array}$ & $\begin{array}{l}\text { Valor } \\
p\end{array}$ & Variable & $\begin{array}{c}\text { F- } \\
\text { Ratio }\end{array}$ & $\begin{array}{c}\text { Eta } \\
\text { cuadrado } \\
\text { parcial }\end{array}$ & $\begin{array}{c}\text { Valor } \\
\text { p }\end{array}$ \\
\hline \multirow{3}{*}{ Edad } & Posición & 0,009 & 0 & 0,926 & \multirow{3}{*}{$\begin{array}{l}\text { Potencia } \\
\text { Máxima Wingate }\end{array}$} & 1,62 & 0,029 & 0,208 \\
\hline & Categoría & 347,429 & 0,863 & $\begin{array}{r}< \\
0,01\end{array}$ & & 37,43 & 0,405 & $<0,01$ \\
\hline & Interacción & 0,254 & 0,005 & 0,616 & & 1,876 & 0,033 & 0,176 \\
\hline \multirow{3}{*}{ Masa Corporal } & Posición & 0,092 & 0,002 & 0,762 & \multirow{3}{*}{$\begin{array}{l}\text { Potencia } \\
\text { Máxima Relativa }\end{array}$} & 1,02 & 0,021 & 0,278 \\
\hline & Categoría & 16,93 & 0,235 & $<0,01$ & & 65,2 & 0,542 & $<0,01$ \\
\hline & Interacción & 0,106 & 0,002 & 0,746 & & 2,016 & 0,035 & 0,161 \\
\hline \multirow{3}{*}{ Talla } & Posición & 5,78 & 0,095 & 0,02 & \multirow{3}{*}{$\begin{array}{l}\text { RPM a los } 5 \\
\text { segundos } \\
\text { Wingate }\end{array}$} & 0,749 & 0,013 & 0,391 \\
\hline & Categoría & 0,784 & 0,014 & 0,38 & & 0,648 & 0,012 & 0,424 \\
\hline & Interacción & 3,1 & 0,053 & 0,084 & & 0,001 & 0 & 0,982 \\
\hline \multirow{3}{*}{ Carrera 30 m } & Posición & 0,055 & 0,01 & 0,815 & \multirow{3}{*}{$\begin{array}{l}\text { Potencia Mínima } \\
\text { Wingate }\end{array}$} & 0,566 & 0,01 & 0,455 \\
\hline & Categoría & 29,39 & 0,348 & $<0,01$ & & 34,015 & 0,382 & $<0,01$ \\
\hline & Interacción & 0,46 & 0,008 & 0,499 & & 0,61 & 0,011 & 0,438 \\
\hline \multirow{3}{*}{$\begin{array}{l}\text { Salto en } \\
\text { contramovimiento }\end{array}$} & Posición & 0,625 & 0,011 & 0,433 & \multirow{3}{*}{$\begin{array}{l}\text { Potencia Mínima } \\
\text { Relativa } \\
\text { Wingate }\end{array}$} & 0,377 & 0,007 & 0,542 \\
\hline & Categoría & 2,308 & 0,04 & 0,134 & & 22,705 & 0,292 & $<0,01$ \\
\hline & Interacción & 0,186 & 0,003 & 0,668 & & 0,427 & 0,008 & 0,516 \\
\hline \multirow{3}{*}{$\begin{array}{l}\text { Salto desde } \\
\text { sentadilla }\end{array}$} & Posición & 0,243 & 0,004 & 0,624 & \multirow{3}{*}{ VMP Sentadilla } & 2,844 & 0,049 & 0,097 \\
\hline & Categoría & 13,855 & 0,201 & $<0,01$ & & 21,416 & 0,28 & $<0,01$ \\
\hline & Interacción & 0,083 & 0 & 0,934 & & 0,021 & 0,093 & 0,021 \\
\hline \multirow{3}{*}{ RM Alcanzado } & Posición & 1,274 & 0,023 & 0,264 & \multirow{3}{*}{ Peso VMP } & 0,048 & 0,001 & 0,828 \\
\hline & Categoría & 139,609 & 0,717 & $<0,01$ & & 11,375 & 0,171 & 0,001 \\
\hline & Interacción & 0,067 & 0,001 & 0,797 & & 0,853 & 0,015 & 0,36 \\
\hline
\end{tabular}

Tabla 2. Resultados en función de las categorías, posiciones de juego y su interacción.

Existe un efecto principal significativo por categorías en la mayoría de pruebas analizadas, destacamos la prueba de velocidad, carrera de 30 metros, F55 $=29,39 ; p<0,01$, eta cuadrado $=0,348$ entre las jugadoras de infantil y pre-juvenil.

Sin embargo, no se encontró un efecto significativo por categorías en la prueba de salto con contra-movimiento, $F 55=2,30 ; p=0,13$, eta cuadrado $=0,04$ entre las jugadoras de infantil y pre-juvenil.

Con respecto a la posición de juego, se encontró un efecto significativo en la variable de talla, $\mathrm{F} 55=5,78 ; \mathrm{p}=0,02$, eta cuadrado=0,095 entre las porterasdefensas y volantes- delanteras. 
Se destaca aquellas variables en que no se encontró un efecto significativo en la posición de juego con respecto a la masa corporal, $F 55=0,092 ; p=0,76$, eta cuadrado $=0,002$.

Se encontró una interacción significativa entre la posición de juego y la categoría en VMP sentadilla, F55 $=21,41 ; p=0,021$, eta cuadrado $=0,093$ entre las jugadoras.

\section{DISCUSIÓN}

En la literatura encontramos un predominio en los estudios que describen las características físicas del rendimiento de la rama masculina de este deporte, superando en gran número a los de la rama femenina (Vescovi et al., 2011; Mujika et al., 2009). La gran mayoría de estos estudios han sido realizados con jugadoras elite y mayores de edad que no se acercan a las características de este estudio. Sin embargo, hallamos algunas investigaciones en mujeres futbolistas con rango de edades bastante amplio que en algunos casos incluyen registros de nuestras categorías y variables analizadas.

Inicialmente en las características antropométricas de masa corporal y talla que nos ocupó en este estudio, queremos mencionar el estudio de Manson et al. (2014), con 52 jóvenes futbolistas elite de Nueva Zelandia, distribuidas en tres categorías por edad, entre los 14 y los 36 años, en la categoría sub 17(14-17 años) reportaron una talla y masa corporal de $164 \mathrm{~cm}( \pm 5,15 \mathrm{~cm})$ y $58,0 \mathrm{~kg}( \pm 5,48 \mathrm{~kg})$, respectivamente. Estos datos en cuanto a la talla y masa corporal son muy similares a los hallados en nuestra categoría pre-juvenil.

Sander et al (2013), con una muestra de 134 jugadoras elite alemanas, en tres categorías desde los 15 hasta los 19 años, el grupo por debajo de 15 años (1315 años) registro una talla y masa corporal de $155,9 \mathrm{~cm}$ y $46,4 \mathrm{~kg}$ respectivamente, siendo ambos datos inferiores a los nuestros. Cabe anotar que fueron los datos iniciales, ya que este estudio duro dos años.

Nicolao, et al. (2010) con 36 jugadoras brasileras entre los 12 y 15 años, en un estudio cuyo objetivo fue la influencia de la maduración sexual en el umbral del lactato en jugadoras de fútbol. En relación con la masa corporal y talla, reportaron en la edad de 13,17 años $( \pm 1,16$ años) un peso de $49,33 \mathrm{~kg}( \pm 7,47 \mathrm{~kg})$ y una talla de $155,67 \mathrm{~cm}( \pm 5,01 \mathrm{~cm})$ resultados muy similares a los nuestros en la categoría infantil.

En relación con la velocidad de desplazamiento existen en la literatura gran número de estudios (Spencer et al (2005); Polman et al (2004), Vescovi y Mcguigan (2008) que han realizado pruebas de velocidad con jugadoras de fútbol; Sin embargo, el uso de diferentes protocolos de evaluación limita la comparación. La prueba más usada en los anteriores estudios fue la de $20 \mathrm{~m}$, específicamente con 
un tiempo de $3,31 \mathrm{~s}( \pm 0,11 \mathrm{~s})$ reportados por la selección nacional de Australia (Tumilty y Darby, 1992).

Existe poca información sobre las diferencias en las características de velocidad entre las posiciones de juego. Vescovi et al. (2011) evaluaron las diferencias en las variables de velocidad (Sprint de 10 a 40 yardas) y concluyeron que no había diferencias significativas entre las posiciones de juego, a pesar de la tendencia de las defensas a ser más lentas. La situación anterior se evidencio en nuestra investigación en la categoría infantil donde las porteras y defensas fueron más lentas, caso contrario se observó en nuestra categoría pre-juvenil.

En cuanto a la fuerza explosiva, es la capacidad física más analizada en la mayoría de estudios encontrados, dentro de estos queremos mencionar el estudio de Vescovi et al. (2011), realizado con 414 adolescentes norteamericanas distribuidas a su vez en 10 categorías por año, desde los 12 hasta los 21 años. En este estudio se evaluó la fuerza explosiva con el salto de contra-movimiento encontrando incrementos hasta los 16 años, a partir de esta edad y hasta los 21 años no se hallaron mejoras significativas. Los registros de este salto en las edades de 12 y 13 años fueron de $37,4 \mathrm{~cm}$ muy superiores a los nuestros $(24,6 \mathrm{~cm})$.

Por esta misma línea encontramos el estudio de Haugen et al. (2012) realizado con mujeres noruegas con 194 participantes entre los 15 y los 35 años de edad, de alto nivel de competición, analizaron la velocidad de desplazamiento (prueba de $40 \mathrm{~m}$ y el salto CMJ) por posiciones de juego, entre los resultados más relevantes en cuanto al salto CMJ las menores de 18 años tuvieron un registro superior al nuestro de $27,9 \mathrm{~cm}$ y en la prueba de velocidad de $40 \mathrm{~m}$ obtuvieron 5 , 77 segundos siendo inferior al nuestro, pero cabe anotar que nuestra prueba de velocidad seleccionada fue de $30 \mathrm{~m}$ y nuestras niñas están entre 12 y 14 años. Con relación a las posiciones de juego, las más rápidas y las que mayor salto CMJ registraron fueron las delanteras.

Por otra parte, Sander et al. (2013), con una muestra de 134 jugadoras elite alemanas, en tres categorías desde los 15 hasta los 19 años, realizó un entrenamiento de fuerza una vez por semana durante dos años. Solo este entrenamiento más el entrenamiento normal de fútbol por dos años, genero mejoras en la fuerza máxima y en la velocidad en la prueba de $30 \mathrm{~m}$, como la que se realizó en el presente estudio. De esta prueba el tiempo registrado en la categoría por debajo de los 15 años fue de 4,78 segundos, siendo muy similar a las jugadoras de nuestro estudio 4,75 segundos.

En Suramérica, queremos destacar el estudio realizado con jóvenes brasileras Frazilli et al. (2010), quien evaluó dos categorías, la infantil (14-15 años) y la juvenil (16-17 años), en el cuál aplicaron los dos saltos de nuestro estudio SQJ y CMJ respectivamente, obteniendo mejores resultados que los nuestros (32, $38 \pm 4,12)$ y $(35,56 \pm 3,93)$ en la categoría infantil con diferencia entre 8 a $11 \mathrm{~cm}$ con 
respecto a nuestra muestra en los dos saltos. En la prueba de velocidad seleccionaron la de $20 \mathrm{~m}$ con un promedio de 3 segundos.

Teniendo en cuenta las posiciones de juego, se encontraron estudios con futbolistas adultas entre los 18 y 21 años. Dentro de esta categoría destacamos a Izquierdo et al. (2008) con una muestra de 42 mujeres futbolistas de primera división españolas, sus principales resultados en las variables de fuerza explosiva no aparecieron diferencias altamente significativas por posiciones de juego, y en este caso, es aún más llamativo el resultado tan bajo obtenido por las porteras en comparación con el resto de las jugadoras de otras posiciones. Caso contrario se obtuvo en nuestro estudio, donde las porteras y defensas obtuvieron los mejores registros en la mayoría de variables estudiadas.

Finalmente, Pantelis et al. (2014), con un grupo de jugadoras griegas (54) tomando como variable las posiciones de juego y aplicando la prueba de Wingate, analizo la potencia media, la potencia máxima, y la potencia pico entre otras variables, resaltando la potencia pico con registros en las porteras $(658 \pm 29 \mathrm{~W})$, las mediocampistas $(577 \pm 78 \mathrm{~W})$, las defensas $(570 \pm 63 \mathrm{~W})$ y finalmente las delanteras $(571 \pm 85 \mathrm{~W})$.

El único estudio realizado en Colombia de acuerdo con nuestra revisión bibliográfica fue el de Méndez et al. (2007) con jóvenes hombres entre 14 y 20 años, trabajo donde se valoró la fuerza explosiva con los mismos saltos de este estudio CMJ Y SQJ, y la velocidad con la prueba de $20 \mathrm{~m}$. Su objetivo principal fue comparar el método de $1 \mathrm{RM}$ trabajado al $85 \%$ con otro al $75 \%$ para mejorar la potencia muscular. Por lo tanto, el presente estudio es el primero realizado en Colombia en la rama femenina que caracteriza estas variables.

\section{CONCLUSIONES}

1- De acuerdo con nuestro objetivo general planteado, se encontraron diferencias significativas en la mayoría de variables estudiadas entre categorías y posiciones de juego, a excepción del Salto CMJ, RPM a los $5 \mathrm{~s}$ y peso VMP. Lo anterior puede sugerir, que las anteriores variables no parecen ser indicadores sensibles que permitan distinguir entre los factores agrupados en este estudio.

2- Los resultados revelan la existencia de variaciones muy pequeñas entre las posiciones de juego y sólo las porteras y defensas parecen tener unas características de condición física específica relacionadas con las exigencias técnicas de dichas posiciones de juego. Además, sus registros en la mayoría de variables analizadas, fueron mejores que el resto de las posiciones de juego. 


\section{REFERENCIAS BIBLIOGRÁFICAS}

Andersson, H., Krustrup, P., \& Mohr, M. (2007). Differences in movement pattern, heart rate and fatigue development in international versus national league matches of Swedish and Danish elite female soccer players. Journal of Sports Science and Medicine, 6, (10), 106-110.

Berger, J. (1982). Estimation in continuous exponential families: Bayesian estimation subject to risk restrictions and inadmissibility results. In Statistical Decision Theory and Related Topics, Eds. S. Gupta. New York: Academic Press.

Datson, Naomi ; Hulton, Andrew ; Andersson, Helena ; Lewis, Tracy ; Weston, Matthew ; Drust, Barry \& Gregson, Warren (2014). Applied Physiology of Female Soccer: An Update. Sports Medicine, Vol.44 (9), pp.1225-1240. https://doi.org/10.1007/s40279-014-0199-1

D'ottavio, S. (1998). El entrenamiento de la fuerza en el fútbol: consideraciones generales y medios de control. Training Fútbol, 26: 24-37.

Frazilli, E.H; De arruda, M; Mariano, T; Cossio Bolaños, M.A. (2010). Correlación entre fuerza explosiva y velocidad en jóvenes futbolistas. "Biomecánica: Órgano de la sociedad ibérica de biomecánica y biomateriales", vol. 18, núm. 2, p. 19-24 http://hdl.handle.net/2099/12308

González- Badillo, J.J. (1997). Fundamentos del Entrenamiento de la Fuerza. Aplicación al Alto Rendimiento Deportivo; Madrid; Edit. Inde.

Haugen, Thomas A.; Tønnessen, Espen ; Seiler, Stephen (2012). Speed and countermovement-jump characteristics of elite female soccer players, 19952010. International Journal of Sports Physiology and Performance, Vol.7 (4), pp.340-349. https://doi.org/10.1123/ijspp.7.4.340

Hewitt, A; Withers, R; Lyons, K. (2007). Match analyses of Australian international women soccer players using an athlete-tracking device. J. Sport Sci. Med., 10 Supple: 107.

Impellizzeri, F. M., Rampinini, E., Castagna, C., Bishop, D., Ferrari Bravo, D., Tibaudi, A., \& Wisloff, U. (2008). Validity of a repeated-sprint test for football. Int J Sports Med, 29(11), 899-905. doi: 10.1055/s-2008-1038491

Izquierdo, M., Hakkinen, K., Gonzalez-Badillo, J. J., Ibanez, J., \& Gorostiaga, E. M. (2002). Effects of long-term training specificity on maximal strength and power of the upper and lower extremities in athletes from different sports. European Journal of Applied Physiology, 87(3), 264-271. doi: 10.1007/s00421-0020628-y

Izquierdo, J; Zarzuela, R; Sedano, S; De Benito, A; Salgado, I y Cuadrado, G. (2008). Estudio comparativo de factores antropométricos y físico-técnicos en jóvenes futbolistas de elite de ambos sexos, en función de la posición habitual de juego. V Congreso asociación española de ciencias del deporte. LeónEspaña.

Kotzamanidis, C., Chatzopoulos, D., Michailidis, C., Papaiakovou, G., \& Patikas, D. (2005). The effect of a combined high-intensity strength and speed training program on the running and jumping ability of soccer players. J Strength Cond Res, 19(2), 369-375. doi: 10.1519/r-14944.1 
Manson SA, Brughelli M, Harris NK. (2014). Physiological characteristics of international female soccer players. J Strength Cond Res. 28(2):308-18. https://doi.org/10.1519/JSC.0b013e31829b56b1

Méndez, E; Márquez, J y Castro, C. (2007). El trabajo de fuerza en el desarrollo de la potencia en futbolistas de las divisiones menores de un equipo profesional de futbol. Revista médica Universidad de Antioquia. Vol. 20, n 2, pp 127-143.

Mohr, M; Ellingsgaard, H; Andersson, H; Bangsbo, J; Krustrup, P. (2003). Physical demands in high-level female soccer - application of fitness tests evaluate match performance. En: Book of abstracts World Congress on Science and Football 5. (Editado por Alves, F; Cabri, J; Diniz, J.A; Reilly, T). 37-38. Gymnos Editorial Deportiva: Madrid

Mujika, I., Santisteban, J., Impellizzeri, F. M., \& Castagna, C. (2009). Fitness determinants of success in men's and women's football. J Sports Sci, 27(2), 107-114. doi: 10.1080/02640410802428071

Nicolao, Ala; Pedrinelli, A ; Zogaib, Psm ; Orbetelli, R ; Neto, Tld (2010). Influence of Sexual Maturation in Lactate Threshold in Female Soccer Players. Revista Brasileira De Medicina Do Esporte, Vol.16(5), pp.335-338. https://doi.org/10.1590/S1517-86922010000500003

Pantelis T. Nikolaidis (2014) Physical Fitness in Female Soccer Players by Player Position: A Focus on Anaerobic Power. Human Movement. Volume 15, Issue 2, Pages 74-79. https://doi.org/10.2478/humo-2014-0006

Polman R, Walsh D, Bloomfield J, (2004). Effective conditioning of female soccer players. J Sports Sci. 2004;22(2):191-203. https://doi.org/10.1080/02640410310001641458

Robertson, R. J., Goss, F. L., Rutkowski, J., Lenz, B., Dixon, C., Timmer, J. Andreacci, J. (2003). Concurrent validation of the OMNI perceived exertion scale for resistance exercise. Medicine \&amp Science in Sports \&amp Exercise, 35(2), 333-341. https://doi.org/10.1249/01.MSS.0000048831.15016.2A

Sander A, Keiner M, Wirth K, Schmidtbleicher D. (2013) Influence of a 2-year strength-training programme on power performance in elite youth soccer $\begin{array}{llll}\text { players. } & \text { Eur } J \text { Sport } & \text { Sci. } & \text { 13(5):445-51. }\end{array}$ https://doi.org/10.1080/17461391.2012.742572

Sanchez-Medina L, Gonzalez-Badillo JJ, Perez CE, Pallares JG. (2014). Velocityand power-load relationships of the bench pull vs. bench press exercises. Int J Sports Med. 35(3):209-16. doi:10.1055/s-0033-1351252

Scott, D y Drust, B (2007) Work-rate analysis of elite female soccer players during match-play. Journal of Sports Science and Medicine, Suppl. 10, 106-110.

Spencer M, Bishop D, Dawson B, (2005). Physiological and metabolic responses of repeated-sprint activities: specific to field-based team sports. Sports Med. 35(12):1025-44.https://doi.org/10.2165/00007256-200535120-00003

Stolen T, Chamari K, Castagna C, Wisloff U (2005). Physiology of soccer: an update. Sports Med. 35(6):501-36. https://doi.org/10.2165/00007256-200535060$\underline{00004}$

Suleyman, P., Bekir, M., Mürsel, B., Mustafa, A., Ekrem, B., Çağrı, Ç., \& Mehmet, G. (2010). The effects of the game form training method (7v7 match) on 
strength parameters of soccer players. Bulletin of the Transilvania University of Braşov, 2(51).

Todd, M.K; Scott, D; Chisnall, P.J. (2002) Fitness characteristics of English female soccer players: An analysis by position and playing standard. En: Science and Football IV. Proceedings of the 4th World Congress of Science and Football. (Editado por Spinks, W; Reilly, T; Murphy, A.). 374-81. Routledge: Nueva York.

Tumilty D, y Darby S. (1992). Physiological characteristics of Australian female soccer players. J Sports Sci. 10:145

Vescovi JD, y Mcguigan MR. (2008). Relationships between sprinting, agility, and jump ability in female athletes. J Sports Sci. 26(1):97-107. https://doi.org/10.1080/02640410701348644

Vescovi, J. D.; Rupf, R.; Brown, T. D. \& Marques, M. C. (2011) Physical performance characteristics of high-level female soccer players 12-21 years of age. Scandinavian Journal of Medicine \& Science in Sports, Vol.21 (5), pp.670678. https://doi.org/10.1111//.1600-0838.2009.01081.

Número de citas totales / Total references: 30 (100\%)

Número de citas propias de la revista /Journal's own references: $0(0 \%)$

Rev.int.med.cienc.act.fís.deporte - vol. 19 - número 73 - ISSN: 1577-0354 